\title{
ASSESSING BITE FORCE ESTIMATES IN EXTINCT MAMMALS AND ARCHOSAURS USING PHYLOGENETIC PREDICTIONS
}

\author{
by MANABU SAKAMOTO (D) \\ School of Life Sciences, University of Lincoln, Lincoln, UK; msakamoto@lincoln.ac.uk \\ Typescript received 29 November 2020; accepted in revised form 7 June 2021
}

\begin{abstract}
Bite force is an ecologically important biomechanical performance measure that is informative in inferring the ecology of extinct taxa. However, biomechanical modelling to estimate bite force is associated with some level of uncertainty. Here, I assess the accuracy of bite force estimates in extinct taxa using a Bayesian phylogenetic prediction model. I first fitted a phylogenetic regression model on a training set comprising extant data. The model predicts bite force from body mass and skull width while accounting for differences owing to biting position. The posterior predictive model has a 93\% prediction accuracy as evaluated using leave-one-out cross-validation. I then predicted bite force in 37 species of extinct mammals and archosaurs from the posterior distribution of predictive models, generating posterior predictive
\end{abstract}

distributions of null expectations given body mass, skull width and phylogenetic position. Biomechanically estimated bite forces from the literature fall within the posterior predictive distributions for all except four species of extinct taxa and are thus as accurate as predicted from body size and skull width, given the variation inherent in extant taxa and the amount of time available for variance to accrue. Biomechanical modelling remains a valuable means to estimate bite force in extinct taxa and should be reliably informative of functional performances and serve to provide insights into past ecologies.

Key words: bite force, dinosaur, sabre-toothed cat, phylogenetic comparative method, phylogenetic prediction, regression.
Bite force is the physical output of a musculo-skeletal biomechanical system (Sinclair \& Alexander 1987), comprised of various phenotypic traits (jaw shape, muscle anatomy), and has a tangible physical interaction with the animal's dietary ecology. Therefore, bite force is at the interface of form, function and ecology, and acts as the single-valued biomechanical performance measure that is under positive phenotypic selection (Sakamoto et al. 2019). This means that bite force is the sum product of the morpho-functional adaptations associated with feeding. Crucially, empirical evidence indicates that bite force indeed correlates with ecomorphology across various tetrapod groups (Herrel et al. 2005, 2010; Wroe et al. 2005; Herrel \& O'Reilly 2006; Christiansen \& Wroe 2007; Dumont et al. 2012, 2014), demonstrating its importance in understanding dietary ecology (Anderson et al. 2008).

Bite force is then a convenient proxy of dietary ecology in extinct taxa for which direct evidence of ecology is not always available (Anderson et al. 2008). While bite force may not entirely predict the nuances of dietary ecology, it serves as a useful single-valued measure of morphofunctional adaptations. Biomechanical models involving bite force in one way or another have indeed contributed to various insights into the dietary ecologies of extinct taxa (Rayfield et al. 2001; Rayfield 2004; Wroe et al. 2005; Anderson \& Westneat 2007; McHenry et al. 2007; Slater \& Van Valkenburgh 2009; Bates \& Falkingham 2012; Lautenschlager 2013; Lautenschlager et al. 2016; Gignac \& Erickson 2017).

However, as bite force estimates are sensitive to muscle parameters, the lack of muscle preservation in fossil specimens means that estimating bite force in extinct taxa is associated with an unknown level of uncertainty (Lautenschlager 2013; Bates \& Falkingham 2018). Whether this uncertainty should hinder our abilities to reliably infer biomechanical performances and past ecologies is up for debate and largely depends on the outlook of individual researchers. Crucially, statistical assessments of the accuracy of bite force estimates in extinct taxa has been lacking, thus making the impact of such methodological uncertainties unknown: how severe is this uncertainty in bite force estimation?

Here, I assess the accuracies of bite force estimates in extinct taxa using the posterior predictive distributions of a phylogenetic prediction model (Organ et al. 2007) based on bite force data in extant taxa (Sakamoto et al. 2019), 
accounting for phylogenetic non-independence owing to shared ancestry (Harvey \& Pagel 1991). Given a strong and significant relationship between bite force and predictor variables (e.g. body mass, skull widths) and phylogenetic information, it is possible to predict bite force in extinct taxa using their corresponding predictor variable values (Organ et al. 2007). The posterior predictive distributions from such a model can serve as the null expectations. Bite forces estimated from biomechanical models for extinct taxa found in the literature can then be tested against these posterior predictive distributions. If such biomechanical estimates of bite force fall within the posterior predictive distribution of the model, then those estimates are as accurate as can be expected from extant data.

\section{MATERIAL AND METHOD}

I used a Bayesian phylogenetic prediction model (Organ et al. 2007) to assess biomechanical bite force estimates in extinct taxa. Phylogenetic predictions were made from a multiple regression model of bite force $\left(\log _{10} F_{\text {Bite }}\right)$ against body mass $\left(\log _{10} M_{\text {Body }}\right)$ and skull widths $\left(\log _{10} W_{\text {Skull }}\right)$ in extant amniotes ( $\mathrm{N}=188$; Fig. 1) (Sakamoto et al. 2019), accounting for phylogenetic non-independence of data points owing to shared ancestry (Harvey \& Pagel 1991). I included skull widths along with body mass as predictor variables, because the former has been shown to predict bite force accurately (Herrel et al. 2005; Gignac \& Erickson 2016; Gignac \& O'Brien 2016), and as the goals here are to predict bite force. I also accounted for differences in slopes amongst groups within the data, namely bats and finches (Fig. 1A, B). These two clades show steeper slopes compared to the rest of the sample (Sakamoto et al. 2019). Additionally, I accounted for differences in bite force owing to differences in biting positions, anterior or posterior (Sakamoto et al. 2019).

Phylogenetic predictions involve two steps. First, I fitted and evaluated a phylogenetic regression model on the training set (bite force and predictor variables in extant taxa) using Markov chain Monte Carlo (MCMC). This will produce a posterior distribution of the regression model $m$. I assessed the accuracy of this prediction model using leave-one-out cross-validation (LOOCV). LOOCV was performed by leaving one taxon out of the training set, fitting a model, and then predicting the taxon of interest using the model (Fig. 1C). I evaluated whether the predicted value differed from the observed value by calculating the proportion of the posterior predictive distribution that fell beyond the value of the biomechanical bite force estimate $\left(p_{\mathrm{MCMC}}\right)$. If the biomechanical bite force estimate fell outside of the vast majority of the posterior predictive distribution $(<5 \%$ of the posterior predictive distribution lay beyond the threshold value) (Fig. 1D), then it is deemed that the biomechanical bite force value is significantly different from the posterior predictive distribution $\left(p_{\text {MCMC }}<0.05\right)$. I repeated this procedure for every tip in the phylogenetic tree over three independent MCMC chains each. Overall prediction accuracy of the phylogenetic regression model is then the number of taxa, for which the prediction is different from the observed value, out of the total number of taxa $\mathrm{N}=188$.

I predicted bite force for the extinct taxa of interest from the posterior distribution of $m$ using MCMC, given their body mass, skull widths, biting positions and phylogenetic positions (Fig. 1C). I used a phylogeny with extinct tips inserted in their relevant positions and predictions were made using MCMC so that rates of evolution along the branches leading to these extinct tips conform to Brownian motion. I then evaluated the literature-based biomechanical bite forces (58 estimates over 37 species; Table 1) against the posterior predictive distributions of the predictive models, using the same approach as in LOOCV (Fig. 1D). I used BayesTraits v3.0.2 (Meade \& Pagel 2019) for both model fitting and predicting (data files, BayesTraits commands and BayesTraits output files are available in Sakamoto 2021), and R (R Core Team 2019) for wrangling, pre-processing and post-processing of data and analytical results.

\section{Comparative bite force data}

I used a subset of the bite force data compiled in a previous study (Sakamoto et al. 2019), to include only those with both body mass and skull widths $(n=224$; Appendix S1; Sakamoto 2021). The bulk of the data were collected from the literature. For each taxon, I took the maximum bite force as the taxon-representative bite force (Appendix S1). Literature-based bite force estimates were standardized as follows:

1. Dry skull estimates in carnivores using muscle stress value $\sigma=300 \mathrm{kN}$ (Wroe et al. 2005) were readjusted to reflect $\sigma=370 \mathrm{kN}$ after (Christiansen \& Wroe 2007).

2. Unilateral bite force estimates (Mazzetta et al. 2009; Reichel 2010; Lautenschlager 2013; Lautenschlager et al. 2016) were doubled to reflect bilateral bites, but not unilateral in vivo bite force measurements, which were used unadjusted, as they can often and regularly attain maximal bite force (Thomason et al. 1990).

Dry skull estimates are known to underestimate in vivo bite force measurements as well as bite force estimates derived from muscle architecture data (Thomason 1991). However, this underestimation is nearly isometric with respect to size (Thomason 1991) making it an underestimation by a constant, rather than an allometric scaling 
A

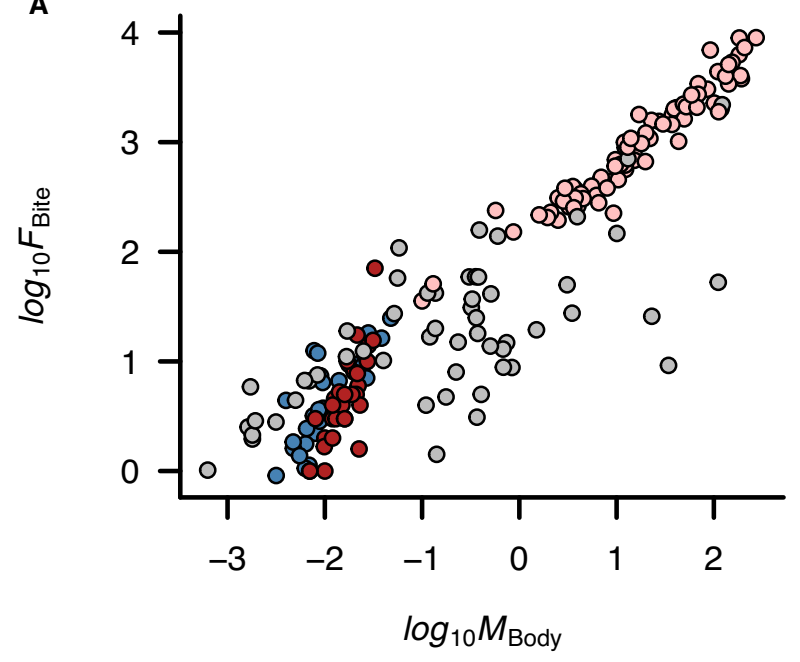

C

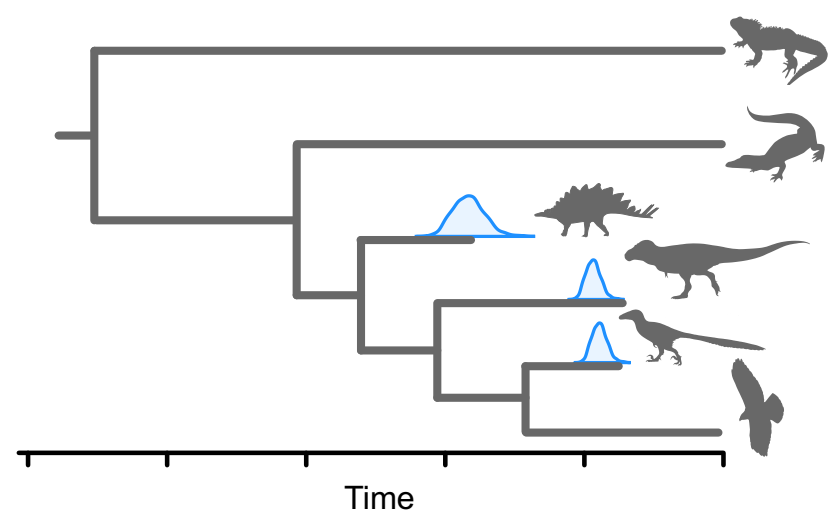

B

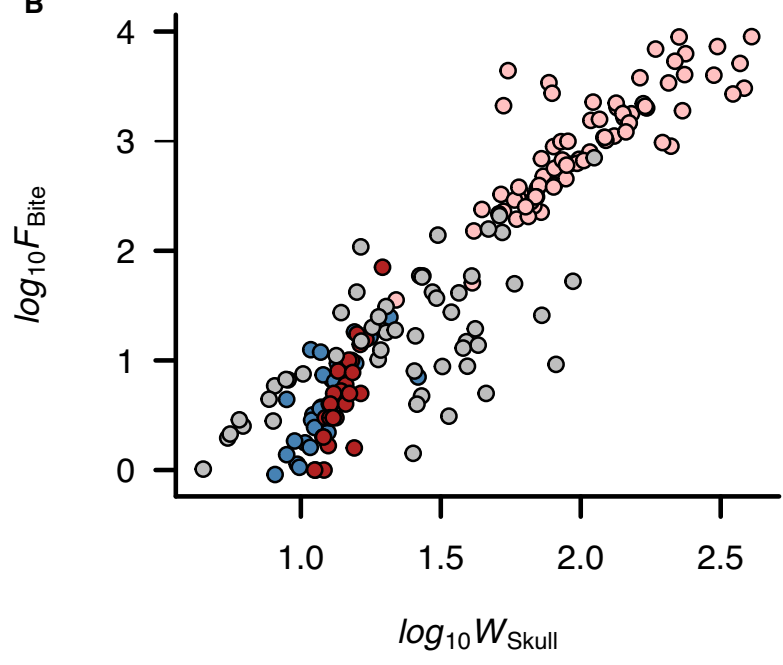

D

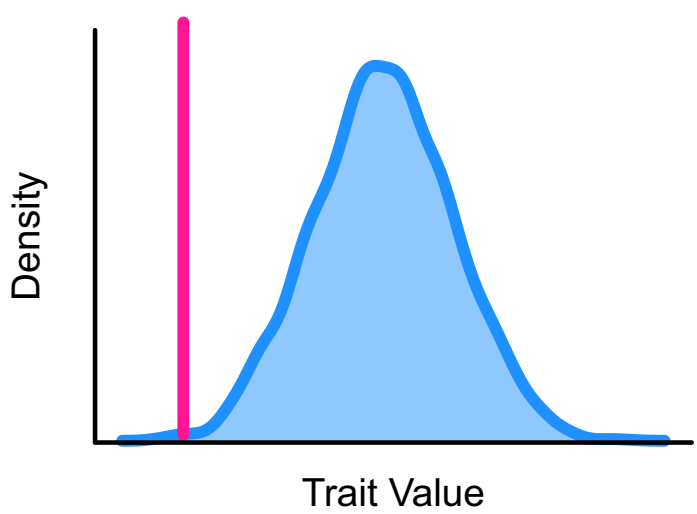

FIG. 1. Phylogenetic predictive modelling uses the relationships between bite force and predictor variables along with the phylogeny to generate posterior predictive distributions for tips in which bite force is unknown. The relationships between bite force and body mass (A) and between bite force and skull width (B) in extant amniotes ( $\mathrm{N}=188)$ are shown, with colours indicating grouping structures (blue, bats; red, finches) and biting positions (grey, anterior; pink, posterior); bite forces for bats and finches are all from anterior positions. C, the relationships in A and B are modelled within a single phylogenetic regression framework and posterior predictive distribution are generated for extinct taxa based on their values of the predictor variables and phylogenetic positions. D, if the observed bite force value (pink vertical line) falls within $95 \%$ of the posterior predictive distribution for any given taxon, then that value is not significantly different from predictions based on predictor variables and its phylogenetic position; if the observed value falls outside of $95 \%$ of the posterior predictive distribution, then that value is significantly different from that predicted from the predictors and its phylogenetic position.

problem. Thus, a simple multiplier is sufficient to adjust for this underestimation, which is the equivalent to using a higher muscle stress value, $\sigma$ (Christiansen \& Wroe 2007). Crucially, previous analyses demonstrate that bite forces estimated by various means (dry skull, multibody dynamics analysis (MDA), finite element analysis (FEA), tooth indentations, tooth fractures) are not significantly different in intercept or slope in a regression framework against body mass compared to those of in vivo measurements (Sakamoto et al. 2019). This means that estimated values are not systematically under-estimating bite force compared to in vivo measurements, nor are there any size-related biases (i.e. where bite force is progressively over/under-estimated in larger taxa).

Maximum bite forces are typically taken at the posteriormost position along the tooth row (i.e. molars in mammals) but are often at more anterior positions, particularly in smaller animals such as lizards, bats and finches, in which standardized biting may be difficult owing to the relative sizes of the bite force transducers (i.e. small animals are incapable of biting comparatively large force plates at their posterior-most biting positions). More importantly, some taxa may have behavioural or morphological constraints for them to bite at anterior positions. Mechanically, 
TABLE 1. Median parameter estimates from the posterior distributions of the predictor variables and $p_{\mathrm{MCMC}}$ values.

\begin{tabular}{llll}
\hline Variable & Parameter & Median estimate & $p_{\text {MCMC }}{ }^{*}$ \\
\hline Intercept & Alpha & 1.515 & 0.000 \\
BitePoint & Beta 1 & 0.144 & 0.115 \\
$W_{\text {Sk }}$ & Beta 2 & 0.230 & 0.050 \\
$W_{\text {Sk_Bats }}$ & Beta 3 & 0.614 & 0.026 \\
$W_{\text {Sk_Finches }}$ & Beta 4 & 3.350 & 0.000 \\
$M_{\text {Body }}$ & Beta 5 & 0.620 & 0.000 \\
$M_{\text {Body_Bats }}$ & Beta 6 & 0.389 & 0.016 \\
$M_{\text {Body_Finches }}$ & Beta 7 & 1.120 & 0.000 \\
\hline
\end{tabular}

*Significant at $<0.05$.

anterior biting positions are lower in magnitude compared to posterior biting positions, so there are concerns that differences in biting positions may bias regression coefficients (higher intercepts for posterior bite forces and slopes likely to differ). In order to take into account such potential biases, each bite force record was categorized into one of two biting position categories (bite points): anterior and posterior. Mid-jaw bite points were categorized as 'anterior' since anterior biting positions are often defined differently across studies; for instance, the biting point at which the tooth comes into contact with food/force transducers first (e.g. caniniform teeth instead of the more anterior incisiform teeth).

\section{Body mass data}

Body mass data are also from a previous study (Sakamoto et al. 2019), collected primarily from the literature, prioritizing values associated with bite force records; especially for individual records in which bite force and body mass were collected for the same individual (Appendix S1; Sakamoto 2021). For bite force records in which associated body mass data were not available, taxonrepresentative body mass data were taken from widely used resources including (Dunning 2007) for birds and (Jones et al. 2009) for mammals (see Appendix S1 for additional sources). For extinct species, I either relied on published estimates (e.g. Henderson \& Snively 2004) or predicted from cranio-dental measurements and published regression equations (Van Valkenburgh 1990) (Appendix S1; Sakamoto 2021). For species in which there are multiple body mass data available, I took the mean value as the taxon-representative body mass.

\section{Phylogeny}

Similarly I used an informal supertree of amniotes based on the Time Tree of Life (TTOL) (Kumar et al. 2017) with fossil tips inserted manually at the appropriate phylogenetic locations (Sakamoto et al. 2019). Divergence times for fossil branches are based on first appearance dates (FAD) with terminal tips extended to their last appearance dates (LAD). I used the full range of temporal durations to scale the branches, as this allows for the maximum amount of time possible for trait evolution to occur (Sakamoto et al. 2019).

\section{RESULTS}

The phylogenetic regression model on the training set explains a high proportion of variance in bite force $\left(R^{2}=0.826\right) . M_{\text {Body }}$ is a significant predictor in all three groups (Table 1$)$. On the other hand, skull width $\left(W_{\mathrm{Sk}}\right)$ is a significant predictor variable in bats and finches, but not in other taxa (Table 1). The effect of bite point is not significant in this model $\left(p_{\mathrm{MCMC}}=0.115\right)$ but I include it here for subsequent predictions as this variable had significant effect in a prior study (Sakamoto et al. 2019).

LOOCV reveals a $92.6 \%$ overall prediction accuracy for the posterior predictive model. In only 14 tips were observed values significantly different from their respective posterior predictive distributions at $p_{\mathrm{MCMC}}<0.05$ (Fig. 2; Table S1). These are: the jaguar, Panthera onca; the aardwolf, Proteles cristatus; 11 species of finches (including five species of Darwin's finches: Geospiza scandens, G. magnirostris, G. fuliginosa, Cactospiza pallida, Platyspiza crassirostris); and the monk parakeet, Myiopsitta monachus.

Out of the 37 extinct taxa, 4 had biomechanical bite force estimates that are significantly different from the posterior predictive distributions (Table S2; Fig. 3) of the phylogenetic regression model based on extant data. These are: the sabre-toothed cats, Xenosmilus hodsonae and Metailurus parvulus; the sauropodomorph dinosaur, Plateosaurus engelhardti; and the ornithischian dinosaur, Stegosaurus stenops (Figs 2, 3). These taxa display bite forces that are significantly lower expected given their body sizes, skull widths and Brownian motion evolution (Fig. 4).

\section{DISCUSSION}

\section{Posterior predictive model}

Overall, the posterior predictive model performs very well in predicting bite force in extant taxa (92.6\% accuracy). In most taxa, bite force is as expected for their body size and skull width, under Brownian motion evolution. That is, changes in residual bite force are proportional to time and do not generally exceed expected amount of changes 


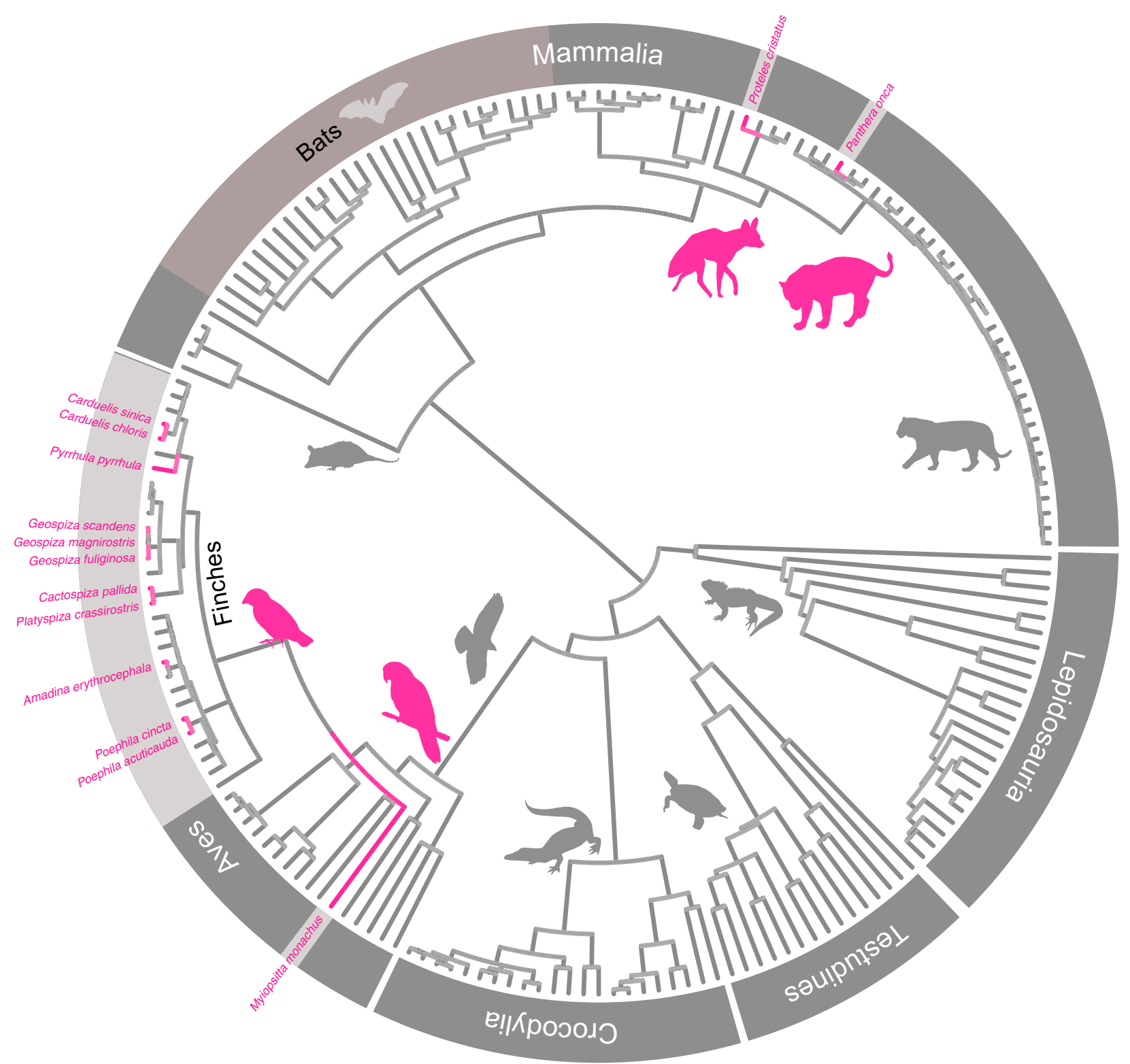

FIG. 2. Phylogeny of extant amniotes $(\mathrm{N}=188)$ showing tips for which the observed bite force values are significantly different from the posterior predictive distributions $\left(p_{\mathrm{MCMC}}<0.05\right.$; pink). Silhouettes from PhyloPic (http://phylopic.org): Geospiza fuliginosa, Manabu Sakamoto, CC-BY 3.0; Psittacid, Amazona aestiva, Ferran-Sayol, CC0 1.0; Panthera onca, Manabu Sakamoto, CC-BY 3.0; Proteles cristatus, Margot Michaud, CC0 1.0; Panthera tigris, Sarah Werning, CC-BY 3.0; Chiroptera, Yan Wong, CC0 1.0; Sphenodon punctatus, Steven Traver, CC0 1.0; Didelphis virginiana, Sarah Werning, CC-BY 3.0; Crocodylia, B. Kimmel, Public Domain Mark 1.0; Buteo buteo, Lauren Anderson, Public Domain Mark 1.0; Chrysemys picta, uncredited, Public Domain Mark 1.0.

along the branches of the phylogenetic tree. Thus, the posterior predictive model can be used to predict bite force in extinct taxa, bracketed by extant taxa on the phylogenetic tree.

The only exceptions are in 14 taxa in which the observed bite forces are significantly different from the posterior predictive distributions $\left(p_{\mathrm{MCMC}}<0.05\right.$; Fig. 2 ; Table S1). These taxa were previously found to have undergone exceptional increases in rates of bite force evolution (Sakamoto et al. 2019), indicative of positive phenotypic selection (Baker et al. 2016) on bite force. Finches in particular radiated rapidly to fill disparate ecological niches (Price et al. 1984; Schluter \& Grant 1984) and that their bite forces significantly deviate from those expected under Brownian motion is strongly reflective of such evolutionary processes. Jaguars are known to have more robust skulls compared to cats of similar sizes (e.g. the leopard) and have extremely high bite forces that 


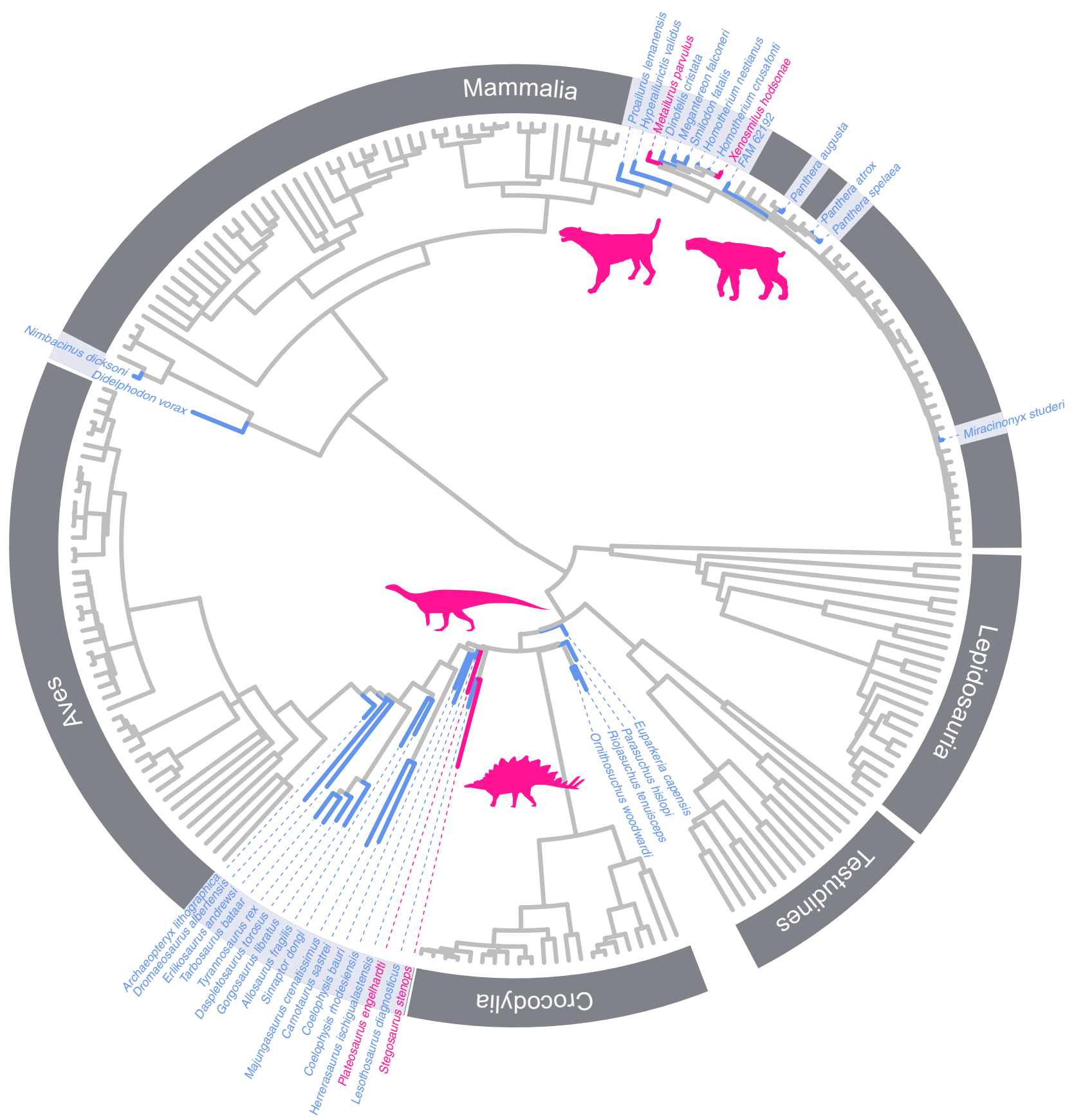

FIG. 3. Phylogeny of amniotes showing extinct tips for which posterior predictive distributions were generated (blue). Taxa for which observed bite forces are significantly different $\left(p_{\mathrm{MCMC}}<0.05\right)$ from their respective posterior predictive distributions are highlighted (pink): Metailurus; Xenoximulus; Plateosaurus; and Stegosaurus. Silhouettes from PhyloPic (http://phylopic.org): Metailurus major, Zimices, CC-BY 3.0; Homotherium, Dantheman9758 (vectorized by T. Michael Keesey), CC-BY 3.0; Plateosaurus, Andrew Knight, CC-BY 3.0; Stegosaurus, Andrew A. Farke, CC-BY 3.0.

enable them to take on large prey. The aardwolf has extremely low bite force compared to its osteophagous relatives and this outlier status within its own family is reflected in its significant departure in bite force from expectations under Brownian motion. The monk parakeet is the only psittaciform in this dataset and its bite force clearly is not as expected given bite forces of other closely related birds (Fig. 2). Thus, all significant departures from the posterior predictive distributions are consistent with our prior understanding of this dataset. 


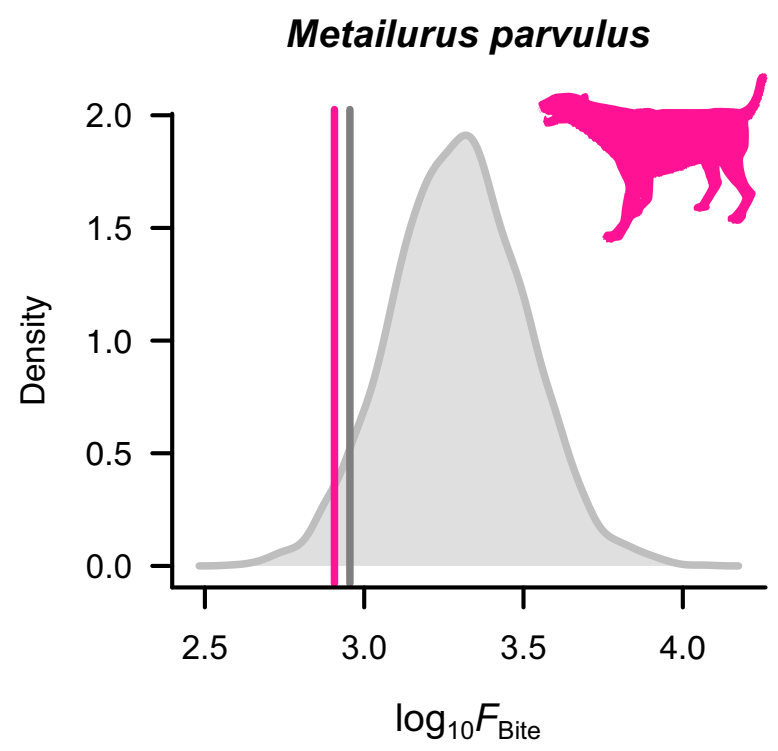

Plateosaurus engelhardti

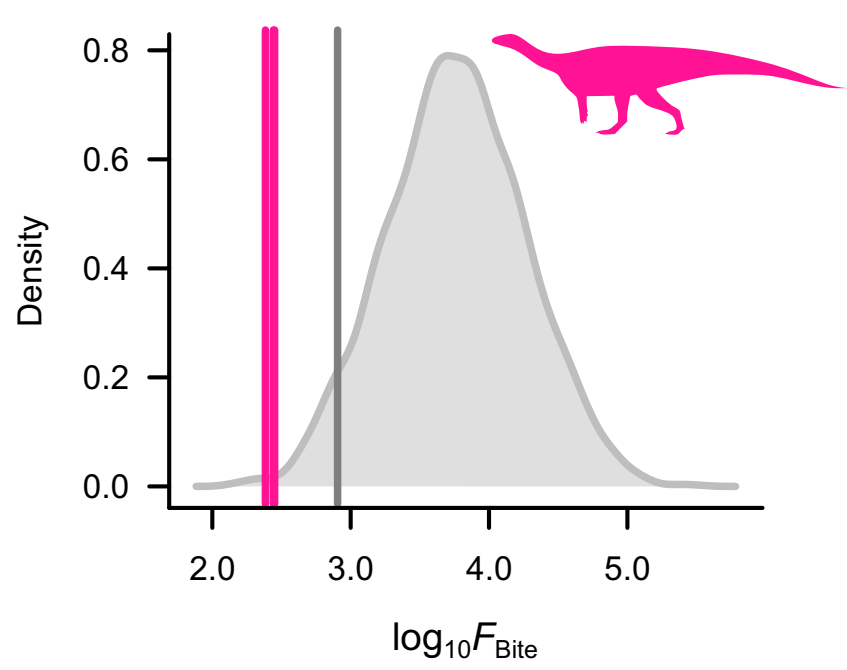

Xenosmilus hodsonae

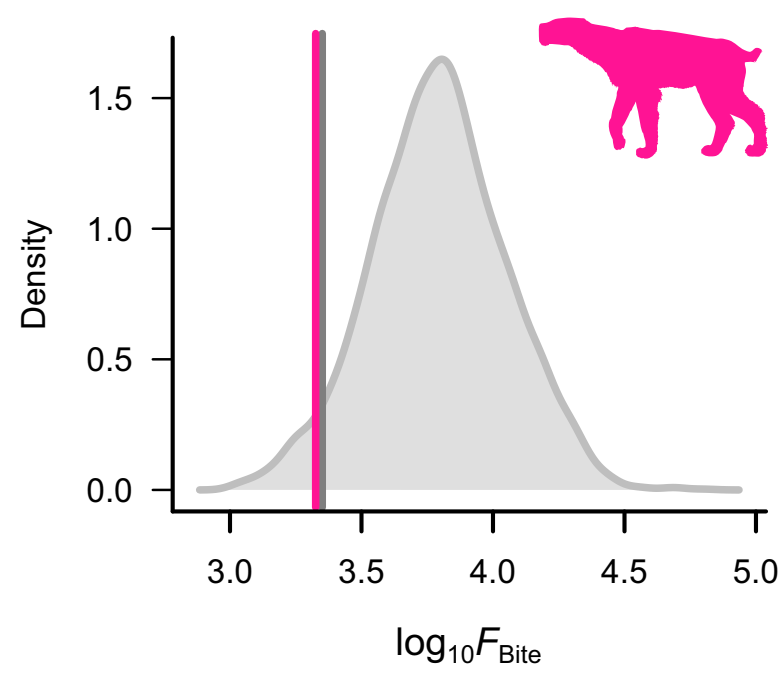

Stegosaurus stenops

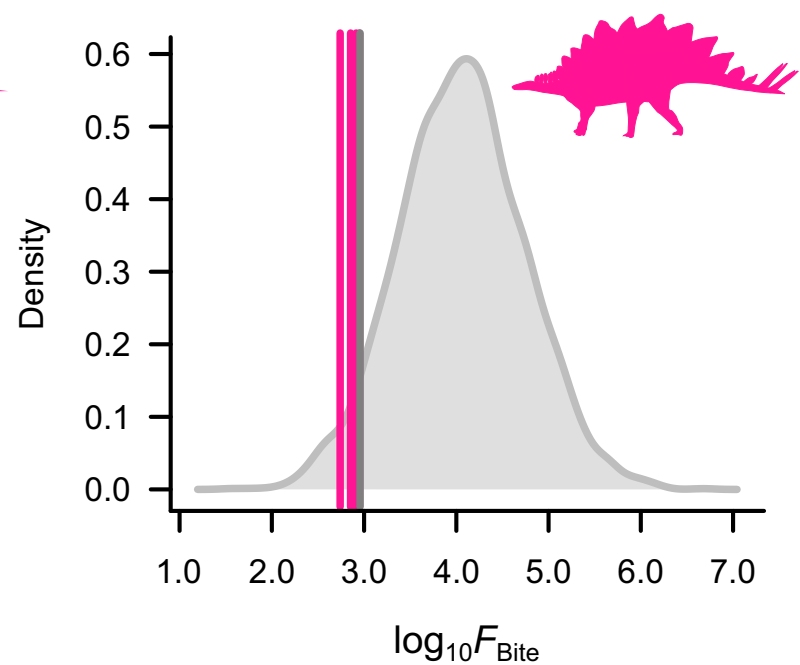

FIG. 4. Posterior predictive distributions of bite force in the four extinct species in which observed bite forces are significantly different. The threshold where $<5 \%$ of the predictions are in the posterior predictive distribution is indicated by the grey vertical line while the observed bite forces available from the literature are shown as pink vertical lines. Silhouettes from PhyloPic (http://phylopic.org): Metailurus major, Zimices, CC-BY 3.0; Homotherium, Dantheman9758 (vectorized by T. Michael Keesey), CC-BY 3.0; Plateosaurus, Andrew Knight, CC-BY 3.0; Stegosaurus, Andrew A. Farke, CC-BY 3.0.

\section{Accuracy of bite force estimates in extinct taxa}

Bite forces in extinct taxa estimated using biomechanical modelling, and currently available in the literature, are generally as accurate as bite forces predicted from the extant relationship between bite force and body size + skull width under Brownian motion evolution accounting for biting position. That is, bite forces estimated from biomechanical approaches in extinct taxa mostly fall within the expected range of variance for their body and skull sizes, given the variation inherent in extant data and the amount of time available for variance to accrue along the branches of the phylogenetic tree.

While the effects of accurate muscle reconstructions were highlighted by Bates \& Falkingham (2018) as a major source of discrepancies in bite force estimates (e.g. in T. rex between authors Bates \& Falkingham 2012 and Gignac \& Erickson 2017), I demonstrate here that such 


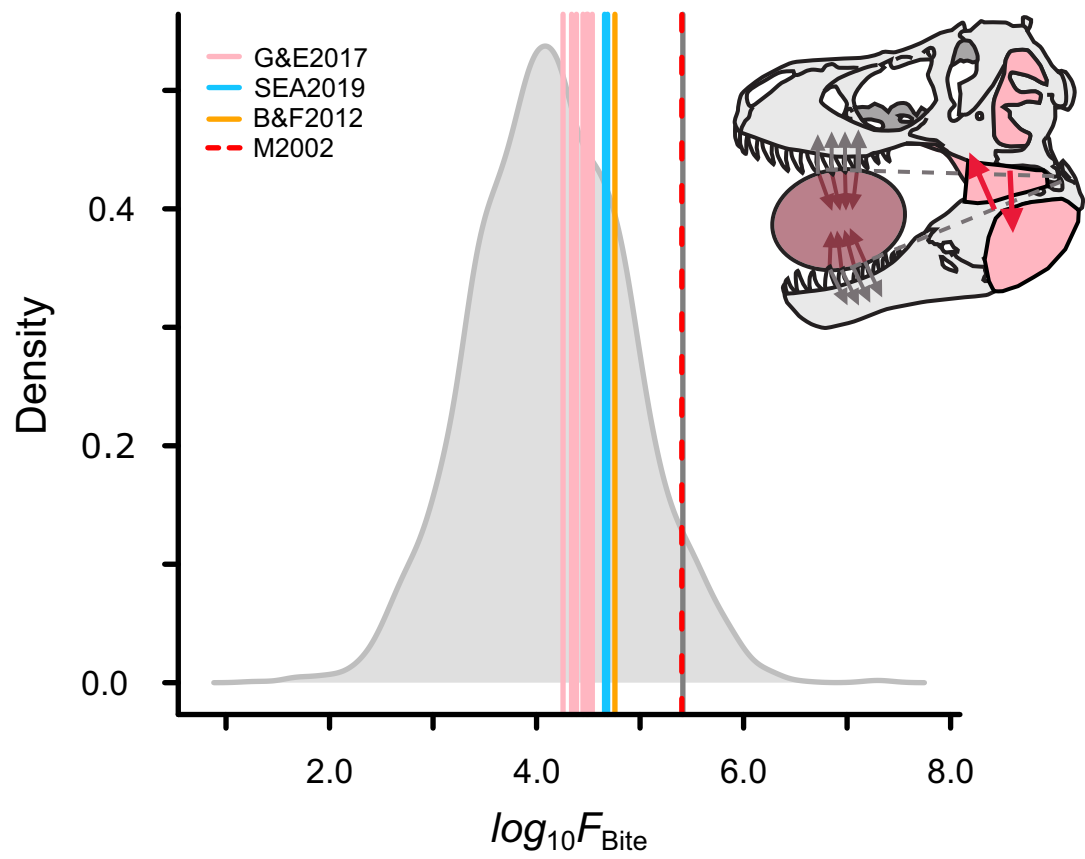

FIG. 5. Biomechanical estimates of $\log _{10}$ bite force for Tyrannosaurus rex mostly fall within the posterior predictive distribution generated from a phylogenetic predictive model. Differences between studies are not statistically significant (pink, Gignac \& Erickson 2017; blue, Sakamoto et al. 2019; orange, Bates \& Falkingham 2012). Bite force extrapolated from a regression model (Meers 2002) (red dashed line) sits on the threshold (solid dark grey line) at $p_{\text {MCMC }}=0.05$. differences are mostly negligible in a phylogenetic comparative context. At least, the variation between authors or force-generating parameterizations generally fall within expected range of variance (Fig. 5). In particular, biomechanical bite force estimates for T. rex (Bates \& Falkingham 2012; Gignac \& Erickson 2017; Sakamoto et al. 2019) all fall within the bulk of the posterior predictive distribution (Fig. 5).

Interestingly, it can be shown here that a nonbiomechanical bite force estimate for $T$. rex based on extrapolation of a non-phylogenetic regression model on extant data (Meers 2002) is most likely to be an overestimate $\left(p_{\mathrm{MCMC}}=0.05\right)$. Meers's estimate (Meers 2002) is higher in value than approximately $95 \%$ of the posterior predictive distribution. This is perhaps unsurprising as the extrapolated bite force of $253123 \mathrm{~N}$ is five times higher than even the highest of the biomechanical estimates (57 $000 \mathrm{~N}$; Bates \& Falkingham 2012), and would require unfeasible muscle volumes and physiological cross sectional areas.

There are however exceptions to the above. Firstly, the two sabre-toothed cats, Xenosmilus and Metailurus have significantly lower bite force estimates than expected (Figs 2, 3). Similar to the case with the extant outliers, these are entirely consistent with our prior understanding of sabre-toothed biting biomechanics (McHenry et al. 2007; Sakamoto et al. 2010). Sabre-toothed cats are known to have smaller jaw closing muscles compared to cats of similar sizes and have been regarded as having weaker bite forces (Wroe et al. 2005; McHenry et al. 2007). Indeed, bite force estimates for most sabre-toothed cats in this dataset generally fall on the lower side of the posterior predictive distributions (Appendix S1). While Sakamoto et al. (2019) did not find evidence for exceptional rates of bite force evolution in sabre-toothed cats using a strict threshold ( $>95 \%$ of rate-scaled trees and twice the background evolution; Baker et al. 2016), they did find some evidence for elevated rates in the family Felidae, including extant conical-toothed cats, under a more relaxed threshold ( $>50 \%$ of rate scaled-trees). As departures from Brownian motion are here gauged using a LOOCV approach using one extinct taxon at a time, the sensitivity to detect significant departures $\left(p_{\mathrm{MCMC}}<\right.$ 0.05 ) may be different compared to the more flexible variable-rates (VR) model (Venditti et al. 2011; Baker et al. 2016) using the entire dataset of extant and extinct data. That is, once the entire range of variation is modelled, then individual departures may not stand out as exceptional rate-increases in the context of a clade exhibiting high variability in trait value. Interestingly, Metailurus has a superficially Panthera-like skull morphology, but its bite force is more reflective of sabre-toothed cats. Metailurus has additional biting functional morphology in line with sabre-toothed cats, such as a wider snout and larger carnassials (Sakamoto \& Ruta 2012).

Secondly, the two herbivorous dinosaurs, Plateosaurus and Stegosaurus have significantly lower bite forces compared to their respective posterior predictive distributions, regardless of force-generating parameter values and ranges chosen by respective authors. These departures from Brownian motion are consistent with previous findings that these two taxa underwent exceptional levels of 
rate-increases (Sakamoto et al. 2019). Given that the effect of skull width is negligible in the phylogenetic regression model employed here (Table 1), the extremely small sizes of the skulls of Plateosaurus and Stegosaurus are probably not accounted for in the predictive model, and thus these taxa appear to have exceptionally low bite forces for their body sizes. As bite force estimates for herbivorous dinosaurs in general are lacking in biomechanical studies, it is difficult to say whether these extremely low values are unique to these taxa or more widespread amongst herbivorous dinosaurs.

Although the default interpretations for such outliers would be to treat them as erroneous estimates, given that outliers in extant taxa determined using LOOCV are consistently those that are known to have extreme bite forces, it is highly likely that the same is true for the extinct taxa identified here as outliers. This is especially so given the uniqueness of the outlying extinct taxa (sabre-toothed cats and herbivorous dinosaurs with extremely small heads).

\section{Bite force and ecological adaptations}

For the most part, bite force can be explained well by body size and skull width. Bite force is known to scale strongly with body size (Sakamoto et al. 2019) as well as skull width (Herrel et al. 2005; Gignac \& Erickson 2016; Gignac \& O'Brien 2016). Skull width in particular is associated with muscle cross-sectional areas, perhaps the most influential determinant of bite force. Thus, the fact that, after accounting for these two influential variables, bite force estimates in the majority of both extant and extinct taxa fall within the expected range of residuals, offers confidence in the reliability of biomechanical methods to estimate bite force. That is, natural selection on bite force is tightly linked with body size and muscle size, and less so with residual variation. The ecological performance of bite force is predominantly associated with ecological niches dictated by size-classes. On the other hand, this means that bite force is a reliable metric for such ecologically meaningful size-classes. This is especially useful for biomechanical modelling of extinct taxa where bite force is applied as a loading parameter or simultaneously estimated.

It follows then, that outliers based on phylogenetic predictive modelling are atypical for their body size, skull width and phylogeny (Fig. 2). As outliers detected here have previously been associated with elevated rates of bite force evolution (Sakamoto et al. 2019), changes in bite force along these branches are in excess to those expected under Brownian motion evolution. Elevated rates are typically taken as evidence for positive phenotypic selection (Baker et al. 2016), but as all extinct outliers have extraordinarily low bite forces, it is more likely that selection acted on phenotypic traits that trade off with bite force. This would be gape (and clearance for hypertrophied upper canines) in sabre-toothed cats and perhaps neck elongation in Plateosaurus and Stegosaurus (Mateus et al. 2009; Maidment et al. 2015), which may be associated with decreases in head size.

\section{CONCLUSION}

Bite force estimates in the majority of extinct taxa examined here fall within their respective posterior predictive distributions generated from a phylogenetic predictive model under Brownian motion evolution. Any discrepancies owing to uncertainties only result in deviations that are fully within the expected range of variance. On the other hand, in both extant and extinct taxa, bite force estimates are only significantly different from their respective posterior predictive distributions when such taxa are already known to have exceptionally high or low bite forces. These results combined indicate that biomechanical bite force estimates are reliable indicators/reconstructions of functional and biomechanical performances in life. This is particularly the case in the context of comparative macro-evolutionary biomechanical analyses (e.g. Sakamoto et al. 2010, 2019), in which statistical parameters are estimated taking into account underlying evolutionary processes in the variance structure of the data.

Acknowledgements. I would like to thank Chris Venditti for advice and guidance on phylogenetic comparative methods, Andrew Meade for support with software and high-performance cluster, and Tai Kubo for discussions. I thank Philip Cox and Eric Snively for reviewing and improving an earlier draft of this manuscript.

\section{DATA ARCHIVING STATEMENT}

Data for this study are available in the Dryad Digital Repository (https://doi.org/10.5061/dryad.rjdfn2z9t) and the Open Science Framework (https://doi.org/10.17605/OSF.IO/8N4ZG).

Editor. Laura Porro

\section{SUPPORTING INFORMATION}

Additional Supporting Information can be found online in the online version of this article https://doi.org/10.1111/pala.12567):

Figure S1. Posterior predictive distribution for each tip in the LOOCV. Observed values are indicated by the pink vertical lines while, the thresholds for $\mathrm{pMCMC}=0.05$ are indicated by the grey line. 
Figure S2. Posterior predictive distribution for each of the predicted extinct taxon. Observed values are indicated by the pink vertical lines while, the thresholds for pMCMC $=0.05$ are indicated by the grey line.

Appendix S1. Supporting information, including details of model fitting and bite force estimates in extant and extinct taxa (Tables S1 and S2).

Table S3. Complete set of comparative data from Sakamoto et al. (2019). Filtering out entries without skull width values will reduce the data to the subset used in this study.

\section{REFERENCES}

ANDERSON, P. S. L. and WESTNEAT, M. W. 2007. Feeding mechanics and bite force modelling of the skull of Dunkleosteus terrelli, an ancient apex predator. Biology Letters, 3, 76-79.

ANDERSON, R. A., McBRAYER, L. D. and HERREL, A. 2008. Bite force in vertebrates: opportunities and caveats for use of a nonpareil whole-animal performance measure. Biological Journal of the Linnean Society, 93, 709-720.

BAKER, J., MEADE, A., PAGEL, M. and VENDITTI, C. 2016. Positive phenotypic selection inferred from phylogenies. Biological Journal of the Linnean Society, 118, 95-115.

BATES, K. T. and FALKINGHAM, P. L. 2012. Estimating maximum bite performance in Tyrannosaurus rex using multibody dynamics. Biology Letters, 8, 660-664.

_ 2018. The importance of muscle architecture in biomechanical reconstructions of extinct animals: a case study using Tyrannosaurus rex. Journal of Anatomy, 233, 625-635.

CHRISTIANSEN, P. and WROE, S. 2007. Bite forces and evolutionary adaptations to feeding ecology in carnivores. Ecology, 88, 347-358.

DUMONT, E. R., DAVAlOS, L. M., GOLDBERG, A., SANTANA, S. E., REX, K. and VOIGT, C. C. 2012. Morphological innovation, diversification and invasion of a new adaptive zone. Proceedings of the Royal Society B, 279, 1797-1805.

— SAMADEVAM, K., GROSSE, I., WARSI, O. M., BAIRD, B. and DAVALOS, L. M. 2014. Selection for mechanical advantage underlies multiple cranial optima in new world leaf-nosed bats. Evolution, 68, 1436-1449.

DUNNING, J. B. Jr 2007. CRC handbook of avian body masses. CRC Press.

GIGNAC, P. and O'BRIEN, H. 2016. Suchian feeding success at the interface of ontogeny and macroevolution. Integrative \& Comparative Biology, 56, 449-458.

GIGNAC, P. M. and ERICKSON, G. M. 2016. Ontogenetic bite-force modeling of Alligator mississippiensis: implications for dietary transitions in a large-bodied vertebrate and the evolution of crocodylian feeding. Journal of Zoology, 299, 229-238.

_ 2017. The biomechanics behind extreme osteophagy in Tyrannosaurus rex. Scientific Reports, 7, 2012.

HARVEY, P. H. and PAGEL, M. D. 1991. The comparative method in evolutionary biology. Oxford University Press.

HENDERSON, D. M. and SNIVELY, E. 2004. Tyrannosaurus en pointe: allometry minimized rotational inertia of large carnivorous dinosaurs. Proceedings of the Royal Society B, 271, S57-S60.
HERREL, A. and O'REILLY, J. C. 2006. Ontogenetic scaling of bite force in lizards and turtles. Physiological \& Biochemical Zoology, 79, 31-42.

— PODOS, J., HUBER, S. K. and HENDRY, A. P. 2005. Evolution of bite force in Darwin's finches: a key role for head width. Journal of Evolutionary Biology, 18, 669-675.

MOORE, J. A., BREDEWEG, E. M. and NELSON, N. J. 2010. Sexual dimorphism, body size, bite force and male mating success in tuatara. Biological Journal of the Linnean Society, 100, 287-292.

JONES, K. E., BIELBY, J., CARDILLO, M., FRITZ, S. A., O'DELL, J., ORME, C. D. L., SAFI, K., SECHREST, W., BOAKES, E. H., CARBONE, C., CONNOLLY, C., CUTTS, M. J., FOSTER, J. K., GRENYER, R., HABIB, M., PlASTER, C. A., PRICE, S. A., RIGBY, E. A., RIST, J., TEACHER, A., BININDA-EMONDS, O. R. P., GITTLEMAN, J. L., MACE, G. M., PURVIS, A. and MICHENER, W. K. 2009. PanTHERIA: a species-level database of life history, ecology, and geography of extant and recently extinct mammals. Ecology, 90, 2648.

KUMAR, S., STECHER, G., SULESKI, M. and HEDGES, S. B. 2017. TimeTree: a resource for timelines, timetrees, and divergence times. Molecular Biology \& Evolution, 34, $1812-1819$.

LAUTENSCHLAGER, S. 2013. Cranial myology and bite force performance of Erlikosaurus andrewsi: a novel approach for digital muscle reconstructions. Journal of Anatomy, 222, 260-272.

- BRASSEY, C. A., BUtTON, D. J. and BARRETT, P. M. 2016. Decoupled form and function in disparate herbivorous dinosaur clades. Scientific Reports, 6, 26495.

MAIDMENT, S. C. R., BRASSEY, C. and BARRETT, P. M. 2015. The postcranial skeleton of an exceptionally complete individual of the plated dinosaur Stegosaurus stenops (Dinosauria: Thyreophora) from the Upper Jurassic Morrison Formation of Wyoming, U.S.A. PLoS One, 10, e0138352.

MATEUS, O., MAIDMENT, S. C. R. and CHRISTIANSEN, N. A. 2009. A new long-necked 'sauropod-mimic' stegosaur and the evolution of the plated dinosaurs. Proceedings of the Royal Society B, 276, 1815-1821.

MAZZETTA, G. V., Cisilino, A. P., BlAnCO, R. E. and CALVO, N. 2009. Cranial mechanics and functional interpretation of the horned carnivorous dinosaur Carnotaurus sastrei. Journal of Vertebrate Paleontology, 29, 822-830.

McHENRY, C. R., WROE, S., ClAUSEN, P. D., MORENO, K. and CUNNINGHAM, E. 2007. Supermodeled sabercat, predatory behavior in Smilodon fatalis revealed by high-resolution 3D computer simulation. Proceedings of the National Academy of Sciences, 104, 16010-16015.

MEADE, A. and PAGEL, M. 2019. BayesTraits v3.0.2. University of Reading, http://www.evolution.rdg.ac.uk/BayesTraitsV3. 0.2/BayesTraitsV3.0.2.html

MEERS, M. B. 2002. Maximum bite force and prey size of Tyrannosaurus rex and their relationships to the inference of feeding behaviour. Historical Biology, 16, 1-12.

ORGAN, C. L., SHEDLOCK, A. M., MEADE, A., PAGEL, M. and EDWARDS, S. V. 2007. Origin of avian genome 
size and structure in non-avian dinosaurs. Nature, 446, 180184.

PRICE, T. D., GRANT, P. R., GIBBS, H. L. and BOAG, P. T. 1984. Recurrent patterns of natural selection in a population of Darwin's finches. Nature, 309, 787-789.

R CORE TEAM 2019. R: A language and environment for statistical computing. v.3.6.2. R Foundation for Statistical Computing, Vienna, Austria.

RAYFIELD, E. J. 2004. Cranial mechanics and feeding in Tyrannosaurus rex. Proceedings of the Royal Society B, 271, 1451-1459.

- NORMAN, D. B., HORNER, C. C., HORNER, J. R., SMITH, P. M., THOMASON, J. J. and UPCHURCH, P. 2001. Cranial design and function in a large theropod dinosaur. Nature, 409, 1033-1037.

REICHEL, M. 2010. A model for the bite mechanics in the herbivorous dinosaur Stegosaurus (Ornithischia, Stegosauridae). Swiss Journal of Geosciences, 103, 235-240.

SAKAMOTO, M. 2021. Data from: Assessing bite force estimates in extinct mammals and archosaurs using phylogenetic predictions. Dryad Digital Repository. https://doi.org/10.5061/ dryad.rjdfn2z9t

— and RUTA, M. 2012. Convergence and divergence in the evolution of cat skulls: temporal and spatial patterns of morphological diversity. PLoS One, 7, e39752.

- Lloyd, G. T. and BENTON, M. J. 2010. Phylogenetically structured variance in felid bite force: the role of phylogeny in the evolution of biting performance. Journal of Evolutionary Biology, 23, 463-478.
RUTA, M. and VENDITTI, C. 2019. Extreme and rapid bursts of functional adaptations shape bite force in amniotes. Proceedings of the Royal Society B, 286, 20181932.

SCHLUTER, D. and GRANT, P. R. 1984. Determinants of morphological patterns in communities of Darwin's finches. The American Naturalist, 123, 175-196.

SINCLAIR, A. G. and ALEXANDER, R. M. 1987. Estimates of forces exerted by the jaw muscles of some reptiles. Journal of Zoology, 213, 107-115.

SLATER, G. J. and VAN VALKENBURGH, B. 2009. Allometry and performance: the evolution of skull form and function in felids. Journal of Evolutionary Biology, 22, 2278-2287.

THOMASON, J. J. 1991. Cranial strength in relation to estimated biting forces in some mammals. Canadian Journal of Zoology, 69, 2326-2333.

— RUSSELL, A. P. and MORGELI, M. 1990. Forces of biting, body size, and masticatory muscle tension in the opossum Didelphis virginiana. Canadian Journal of Zoology, 68, 318-324.

VAN VALKENBURGH, B. 1990. Skeletal and dental predictors of body mass in carnivores. 181-205. In DAMUTH, J. and MACFADDEN, B. J. (eds). Body size in mammalian paleobiology: Estimation and biological implications. Cambridge University Press.

Venditti, C., MEADE, A. and PAGEL, M. 2011. Multiple routes to mammalian diversity. Nature, 479, 393-396.

WROE, S., McHENRY, C. and THOMASON, J. 2005. Bite club: comparative bite force in big biting mammals and the prediction of predatory behaviour in fossil taxa. Proceedings of the Royal Society B, 272, 619-625. 